\title{
RESILIENCE ASSESSMENT FOR INTERDEPENDENT WATER SUPPLY SYSTEMS BASED ON A SYSTEM DYNAMICS MODEL
}

\author{
JEWAN RYU \& HEEKYUNG PARK \\ Department of Civil and Environmental Engineering, KAIST, Republic of Korea
}

\begin{abstract}
Recent catastrophic events such as Hurricane Katrina have highlighted the importance of interdependency to result in cascading effect among critical infrastructures. Previous studies investigated and categorized the interdependency of critical infrastructures based on qualitative consideration and case studies. However, it is still necessary to approach this quantitatively. The main purpose of this study is to model the behavior of interdependent infrastructures in urban systems, especially water and energy supply, and suggest management policy for water supply systems to increase its resilience. The relationship between two infrastructures was converted into causal loop diagrams. The system dynamics model considering the interdependency was developed with components and relationship from previous research. The capacity of water supply system and its recovery capacity under disruptive scenarios was assessed as the resilience of the water supply system. The simulation result showed that the order of interdependency has high sensitivity with the variance of resilience. It was also found that enhancing the resilience of the water supply system improved not only the recovery capacity of the water supply system but also of the energy supply system. The most efficient policy leverage was found in the enhancing feedback loop of resource exchange between two infrastructures. Some assumptions in the relationship between components should be formulated with more concrete observation and mathematical consideration in further studies.
\end{abstract}

Keywords: resilience, system dynamics, interdependent infrastructures, water supply management.

\section{INTRODUCTION}

As the infrastructures have been developed and become more complicated, modern society has become a system that constitutes complex networks of interdependent infrastructures. There have been some qualitative considerations to define and classify the interdependency but there is no universal definition or classification yet [1]. Quantitative modeling and simulation are recently performed based on systemic approach such as system dynamics.

Simultaneously, the complexity of infrastructures emphasized the resilience concept in infrastructure management against disruptive events. The interdependency improves or exacerbate the overall performance of the complex system. Previous research issues that tried to predict disruptive events precisely became inappropriate to be applied to the complex and interdependent infrastructures. The idea that no single factor has complete control on infrastructures has emerged and the conventional mathematical methodologies to model the previous prediction models are not suitable to consider the complexity and interdependency [2]. The difficulties in prediction of those disasters are due to many affecting factors including the interdependency which causes cascading effects among infrastructures. Finally, the concept of resilience has been highlighted which means the capacity to recover system performance after any disruptive events.

Focusing on the complexity of infrastructures, the concept of resilience, which was introduced first in 1970s, was emerged for disaster management in infrastructure engineering. Little [3] argued that the mitigating damage to infrastructure and ensuring continuity of service is complicated by the interdependent nature of infrastructure systems [3]. The efforts to define and quantify resilience of infrastructures have been performed. Infrastructure 
management to improve resilience was also studied to recover the function of system fast when a disruptive event occurs.

But not only the literal definition but also the theoretical definition and classification considering the features of interdependencies was implemented. The interdependency was firstly defined as the bidirectional relationship between infrastructures through which the state of each infrastructure is influenced by or correlated to the state of the other [4].

In this paper, the system dynamics model based on water and energy supply infrastructures which are interdependent was constructed. The water supply capacity was selected to indicate the resilience. The model was simulated under disruptive scenarios and the systemic behavior was analyzed.

\section{METHODOLOGY}

System dynamics model describes the causal relationships between components. The fundamental building block is causal arrow in Fig. 1. The causal relationships are expressed by two kinds of arrows which are enhancing and balancing. The enhancing arrow with positive sign means that two components changes in same direction. Whereas, the balancing arrow with negative sign means that two components changes in opposite direction. Causal arrows organized in circle are feedback loop. The aggregation of many causal relationships and feedback loops determine the behavior of whole system. The relationships between components are converted to mathematical formulation in system dynamics software for simulation. Because it is almost impossible to describe all components and their root relationship in concerned system, appropriate system boundary and assumptions are necessary.

The electricity power and water grid systems are simplified to consider only the supplying process. Therefore, it is assumed that both transmission and pumping capacity has their maximum value if there is no disruption, but the net transmission and pumping capacities are decreased with respect to the ratio of disruption. Fig. 2 shows the causal loop diagrams in the model. Two feedback loops in the center indicates the interdependency between water and energy supply infrastructures [5]. They are connected by price and demand of each resource. The inner feedback loop describes the amplification of demands between two infrastructures. Because water supply infrastructure requires electricity for operation and vice versa, the water demand in energy supply infrastructure and energy demand in water supply infrastructure are essential components in the central feedback loop. The water demand for energy supply increases the total water demand and the water supply is also increased to meet the demand. Then the water supply infrastructure requires more electricity for water treatment and pumping. This leads the increase of energy demand for water supply. Then, the total energy demand is increased and the energy supply is increased to meet the demand.

The outer feedback loop describes the relationship of cost and price to supply water and energy [6]. The infrastructures set the price to meet the total demand of each resource and not to store too much remainder. The more expensive water price results in the much water cost in energy supply, and then the total cost in energy supply is also increased. Then the

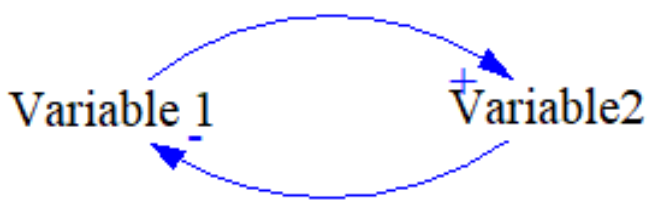

Figure 1: Causal arrows for feedback loop. 


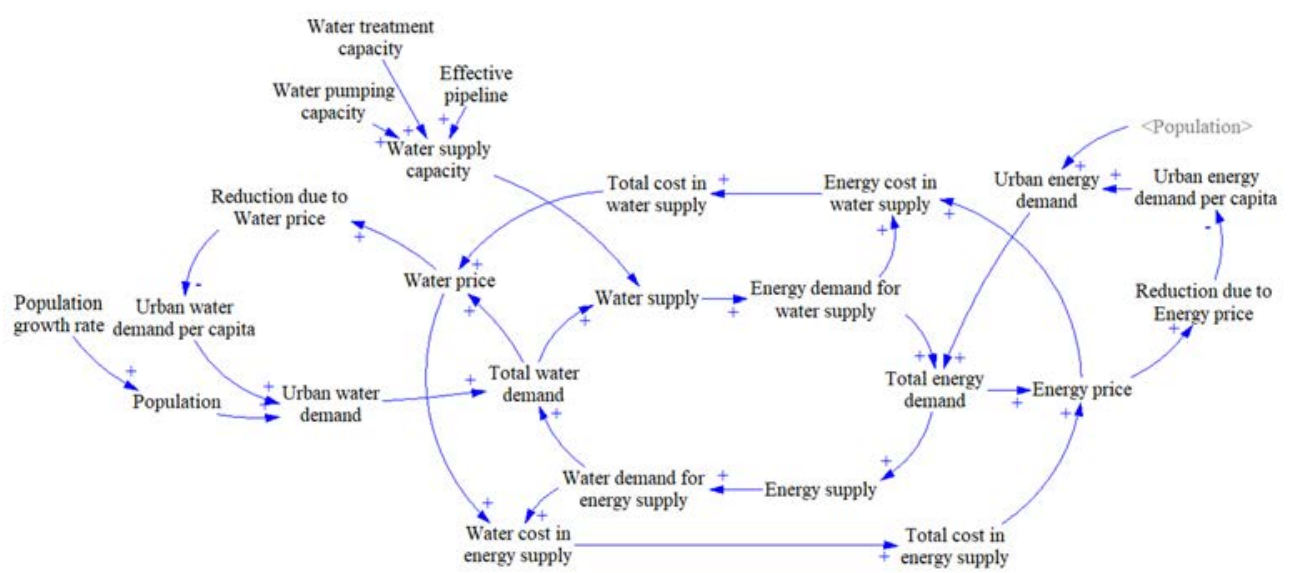

Figure 2: Causal loop diagram of the interdependency between water supply and energy supply system.

energy supply infrastructure set more expensive energy price to afford the increased cost. This leads the energy cost and total cost in water supply infrastructure again. Because causal arrows in central feedback loops are all enhancing arrows, the interdependency in water and energy supply infrastructure will result in continuously increasing demand and cost. But this synergy does not appear in the whole system due to the negative feedback loop in left and right side.

The two feedback loops in left and right side shows the balancing of total resource demand originated in the increased price. The population in urban area and water demand per capita determine the urban water demand. The summation of urban water demand and water demand for energy supply is total water demand and it effects to water price and water supply as described before. The components in this model are summarized in Table 1.

\section{RESULT AND DISCUSSION}

The water resource availability shows a valley form because of the deficit caused by disruption. If there is no disruption, the full resource utility, which means $\int$ resource availability $d t$ for simulation time is $36,000 \cdot$ days. For each scenario, $36,000-\int$ resource availability $d t$ was calculated to measure the resource deficit to show how the people in urban area undergo lack of resources during simulation time. Additionally, the recovery time which means the resource availability returns to normal operation were measured to compare the effect of interdependent critical infrastructures.

To analyze the effect of interdependency between water and energy supply infrastructures, the water availability and recovery time after disruptive events were compared. The water availability measures how the urban area is undergoing lack of water resource so that its recovering graph indicates the resilience. Scenarios in Table 1 were simulated. The both direct and indirect disruptions by each scenario are compared to normal operation.

Comparing scenario from 1 to 3, there exist disruptions in water supply system by cascading effect from electric power infrastructure even though the direct disruption to water supply system is assumed to be zero. The simulation results are shown in Fig. 3(a) and Table 2. The water resource deficit is almost four times larger than normal operation. Moreover, 
Table 1: Simulation conditions.

\begin{tabular}{|l|l|}
\hline No & Description \\
\hline Scenario 1 & Normal operation \\
\hline Scenario 2 & $75 \%$ Energy supply disrupted \\
\hline Scenario 3 & $50 \%$ Energy supply disrupted \\
\hline Scenario 4 & $75 \%$ Water supply disrupted \\
\hline Scenario 5 & $75 \%$ Water supply and energy supply disrupted \\
\hline
\end{tabular}

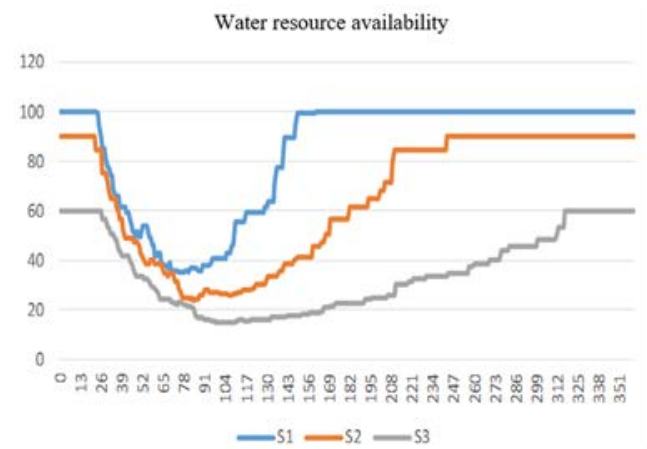

(a)

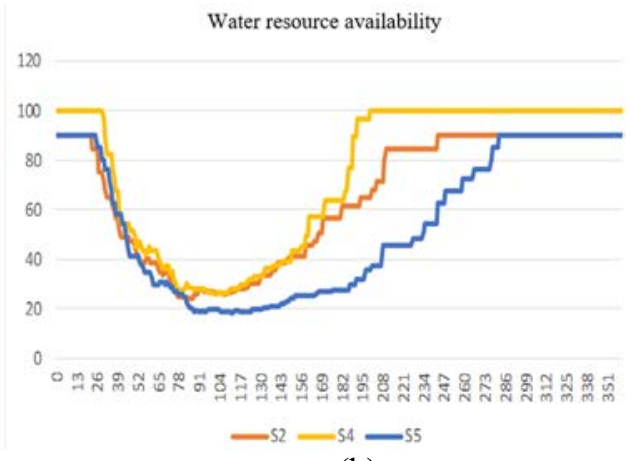

(b)

Figure 3: Effect of different disruptive scale in electric transmission.

Table 2: The resource deficit and recovery time.

\begin{tabular}{|l|c|c|}
\hline No & Total resource deficit $(\% \cdot$ day $)$ & Recovery time (day) \\
\hline Scenario 1 & 5,518 & 170 \\
\hline Scenario 2 & 12,279 & 243 \\
\hline Scenario 3 & 23,204 & 317 \\
\hline Scenario 4 & 8,915 & 231 \\
\hline Scenario 5 & 16,457 & 282 \\
\hline
\end{tabular}

the upper boundary of water resource availability appeared. It was found that the electricity demand at water supply infrastructure becomes larger than the maximum electricity supply capacity after the maximum electricity capacity was deteriorated.

Fig. 3(b) compares the effect of disruption in different interdependent critical infrastructures. Comparing scenario 2 and 4, the cascading effect from electricity to water infrastructures is comparable to the direct damage in water infrastructure in the model. The total deficit was more substantial in scenario 2 than 4 . Obviously, the scenario 5 which is summation of 2 and 4 showed the largest resource deficit and the lowest resource supplying performance. Note that the $50 \%$ limitation in electricity exceeds the $50 \%$ capacity limitation to water supply infrastructure. It is considered that it results from the exponential relationship which were amplified along the causal loops. Another reason is that the maximum transmission capacity generates much lower upper bound in water resource availability. 


\section{CONCLUSION}

After the introduction of interdependency concept for infrastructure engineering, both the qualitative and quantitative researches have been implemented. The first issue was defining the interdependency and then, development of framework to capture interdependency was subsequent effort. But it is still being discussed. Based on the definition and classification of interdependency, researchers modelled the interdependency to figure out cascading effect in modern complex disasters and suggest management policies.

In this work, the disruptive scenarios were applied to give impact in the SD model which describes two interdependent critical infrastructures, focusing on the water supply infrastructure. The interdependency was described with causal loop diagram and converted to stock and flow diagram. Assumptions were introduced to specify the system boundary. Then the model was simulated according to different scenarios in order to compare the effect of interdependency. The mostly deteriorated result showed increase of four times in resource availability deficit and two times in the last recovery time when compared to no disruption case. The simulation result verified the first and second order of cascading effect to deteriorate the performance of water supply infrastructure. It has been confirmed that the damage to infrastructure networked with other infrastructures may cause catastrophic damage to the entire system. Many assumptions were accepted although the result is reasonable with a calibration coefficient. More detailed and qualified relationship among components can be adopted and improve the model.

\section{REFERENCES}

[1] Rinaldi, S.M., Modeling and simulating critical infrastructures and their interdependencies. Proceedings of the 37th Annual Hawaii International Conference on IEEE, p. 8, 2004.

[2] Amin, M., National infrastructures as complex interactive networks. Automation, Control, and Complexity: An Integrated Approach, eds T. Samad \& J. Weyrauch, John Wiley \& Sons, pp. 263-286, 2000.

[3] Little, R.G., Toward more robust infrastructure: observations on improving the resilience and reliability of critical systems. Proceedings of the 36th Annual Hawaii International Conference on System Sciences, 2003.

[4] Rinaldi, S.M., Peerenboom, J.P. \& Kelly, T.K., Identifying, understanding, and analyzing critical infrastructure interdependencies. IEEE Control Systems, 21(6), pp. $11-25,2001$.

[5] Zhuang, Y., A system dynamics approach to integrated water and energy resources management. $\mathrm{PhD}$ thesis, University of South Florida, 2014.

[6] Min, H.S.J., Beyeler, W., Brown, T., Son, Y.J. \& Jones, A.T., Toward modeling and simulation of critical national infrastructure interdependencies. Iie Transactions, 39(1), pp. 57-71, 2007. 